\title{
USO DE TERAPIAS DE MEDICINA ALTERNATIVA Y COMPLEMENTARIA EN LA PROVINCIA DE CORONEL PORTILLO, UCAYALI, PERÚ
}

\author{
Rocío Santiváñez-Acosta (1) 1,a, Félix Valenzuela-Oré (10] 1,2,b, Yolanda Angulo-Bazán (ib) 1,c \\ 1 Centro Nacional de Salud Intercultural, Instituto Nacional de Salud, Lima, Perú. \\ 2 Facultad de Ciencias de la Salud, Universidad Peruana Los Andes, Huancayo, Perú. \\ a Química farmacéutica; ${ }^{\mathrm{b}}$ licenciado en Enfermería, doctor en Salud Pública; ${ }^{\mathrm{c}}$ médica cirujana.
}

\section{RESUMEN}

Se realizó un estudio transversal con el objetivo de evaluar el uso de terapias de medicina alternativa y complementaria (MAC) y sus factores asociados, en la provincia de Coronel Portillo (Ucayali), durante el 2013. Se realizaron 917 encuestas a los jefes de hogar, residentes en tres distritos de la provincia (Callería, Manantay y Yarinacocha), en las que se calculó la proporción de uso de MAC y se analizó la relación con variables sociodemográficas (edad, sexo, grado de instrucción, estado civil, ocupación e ingreso económico). 179 encuestados (19,5\%) mencionaron que utilizaron MAC en los últimos doce meses. Se encontró una relación estadísticamente significativa entre el uso de terapias de MAC y el grado de instrucción $(\mathrm{p}<0,001)$, la ocupación de la persona encuestada $(\mathrm{p}<0,001)$ y su ingreso económico mensual $(\mathrm{p}<0,001)$. Futuras investigaciones definirán las asociaciones encontradas y las variables intervinientes.

Palabras clave: Terapias Complementarias; Estudios Epidemiológicos; Estudios Transversales; Modelos Logísticos; Plantas Medicinales; Acupuntura; Terapia por Acupuntura; Escolaridad; Encuestas y Cuestionarios (Fuente: DeCs BIREME).

\section{USE OF COMPLEMENTARY AND ALTERNATIVE MEDICINE THERAPIES IN THE CORONEL PORTILLO PROVINCE, UCAYALI, PERU}

Citar como: Santiváñez-Acosta R, Valenzuela-Oré F, Angulo-Bazán Y. Uso de terapias de medicina alternativa y complementaria en la provincia de Coronel Portillo, Ucayali, Perú. Rev Peru Med Exp Salud Publica. 2020;37(3):510-5. doi: https://doi. org/10.17843/rpmesp.2020.373.4939

Correspondencia: Rocío Santiváñez Acosta; Av. Defensores del Morro 2268, Lima 9, Perú; roxioxanti@yahoo.com

Recibido: 06/11/2019 Aprobado: 06/05/2020 En línea: 03/07/2020

\begin{abstract}
A cross-sectional study was carried out to evaluate the use of complementary and alternative medicine (CAM) therapies and the factors associated with it, in the province of Coronel Portillo (Ucayali) during 2013. A total of 917 surveys were carried out among household heads in three districts of the province (Callería, Manantay and Yarinacocha), in which the proportion of CAM use was calculated and the relationship with sociodemographic variables (age, sex, educational level, marital status, occupation and income) was analyzed. From the total, 179 respondents (19.5\%) mentioned that they used CAM in the last 12 months. A statistically significant relationship was found between the use of CAM therapies and educational level $(p<0.001)$, respondent's occupation $(p<0.001)$ and monthly income $(p<0.001)$. Future research will define the found associations and the variables involved.
\end{abstract}

Keywords: Complementary Therapies; Epidemiologic Studies; Cross-Sectional Studies; Logistic Models; Plants, Medicinal; Acupuncture; Acupuncture Therapy; Educational Status; Surveys and Questionnaires (Source: MeSH NLM).

\section{INTRODUCCIÓN}

La Organización Mundial de la Salud define a la medicina alternativa y complementaria (MAC) como el conjunto de prácticas de atención sanitaria, que no son parte de la tradición del país (medicina tradicional) y no se encuentran integradas y articuladas totalmente al sistema de salud ${ }^{(1)}$. Estas terapias suelen ofrecer un enfoque holístico y centrado en la persona, lo que hace que la atención en salud integre las esferas biopsicosocial y espiritual de los usuarios ${ }^{(2)}$.

La frecuencia de uso de las terapias de MAC por la población varía en relación al lugar estudiado; sin embargo, en América Latina, a pesar de la aceptación de estas terapias y los fac- 
tores culturales ligados a ellas, solo existen estimaciones de su uso, que calculan que aproximadamente 7 de cada 10 pacientes latinos recurren a terapias no convencionales, como el uso de plantas medicinales para el cuidado de su salud ${ }^{(3)}$. Por otro lado, los determinantes sociales y culturales que influyen en los pacientes para usar terapias de MAC o medicina tradicional son diferentes entre poblaciones que habitan zonas urbanas y zonas rurales ${ }^{(4)}$. Un estudio previo estimó que pertenecer a una etnia indígena constituía un factor significativo en la decisión de usar o no terapias de MAC ${ }^{(5)}$.

En el Perú, la información sobre el uso de estas terapias es bastante limitada. En un estudio realizado en pacientes de consulta externa de Lima Metropolitana se estimó un 29,6\% de uso de terapias de MAC ${ }^{(6)}$; no se han encontrado estudios similares en la Amazonía del Perú, selva urbana o rural.

Ucayali, por sus indicadores de salud en ciertas enfermedades no trasmisibles, es una de las regiones de la Amazonía de mayor importancia, por ejemplo, reporta la mayor concentración de casos notificados de diabetes ${ }^{(7)}$; dolencias en que la MAC cuenta con la mejor evidencia disponible de seguridad y eficacia. Por otro lado, la provincia de Coronel Portillo concentra el 77,3\% de la población de la región Ucayali, los tres distritos estudiados representan el $88 \%$ de la población de la provincia ${ }^{(8)}$ y se dedican mayormente al comercio minoritario: agricultura, ganadería, caza y silvicultura, con presencia importante de población indígena ${ }^{(9)}$. En tal sentido, el objetivo del presente estudio fue evaluar el uso de terapias de MAC y sus factores sociodemograficos asociados, en la provincia de Coronel Portillo durante el 2013.

\section{EL ESTUDIO}

\section{Diseño y población}

Se realizó un estudio transversal analítico, de mayo a diciembre del 2013, en tres distritos: Callería, Manantay y Yarinacocha, de la provincia de Coronel Portillo en Ucayali. Se incluyó a personas con edad mayor o igual a 18 años, domiciliadas en los tres distritos, y se consideró la participación de un miembro por familia (jefe de hogar o quien hiciera sus veces al momento de la encuesta). Se excluyó a las personas que no deseaban participar en el estudio, que no supieran leer o escribir, o que tuvieran problemas para comprender las preguntas de la encuesta.

Se realizó un muestreo aleatorio simple, con distribución proporcional a la población de estudio y de aplicación a todas las viviendas en las manzanas seleccionadas. Se consideró un total de 3394 manzanas (mzs.) que comprenden los distritos de Callería (1367 mzs.), Manantay (990 mzs.), Yarinacocha (1037 mzs.), y un total de 67156 viviendas (Callería: 31575 viviendas, Yarinacocha: 20071 viviendas y Manantay: 15510 viviendas).

\section{MENSAJES CLAVE}

Motivación para realizar el estudio: En el Perú, se ha estudiado poco el uso de la medicina alternativa y complementaria (MAC), considerando que es un país pluricultural, en el que estas prácticas se encuentran arraigadas en la sociedad y se utilizan de forma cotidiana y paralela a la oferta del sistema de salud convencional.

Principales hallazgos: En el ámbito de la Amazonía peruana, en la provincia de Coronel Portillo (Ucayali), se encuestaron a 917 personas, donde se encontró un porcentaje de uso de MAC de 19,5\%.

Implicancias: Estos resultados apoyan la integración de estas terapias al sistema nacional de salud.

Para obtener el tamaño muestral, se consideró una proporción de uso de MAC del 50\%; un nivel de confianza del $95 \%$ y una precisión del $5 \%$. Adicionalmente, se consideró un $10 \%$ de tasa de no respuesta y se obtuvo un tamaño final de 840 encuestas (395 encuestas en Callería, 252 en Yarinacocha y 193 en Manantay). La selección de las viviendas por encuestar se realizó mediante el programa Epidat versión 3.1.

\section{Variables del estudio}

Se consideró como variable dependiente al uso de terapias de MAC en los últimos doce meses, definido como la respuesta afirmativa a la pregunta: $i$ Ha recibido alguna terapia de medicina alternativa y complementaria en los últimos doce meses? En quienes se obtuvo una respuesta afirmativa, se caracterizó el uso de terapias de MAC: tipo de terapia utilizada, institución que provee el servicio y si se pone en conocimiento del médico tratante.

Se evaluaron como posibles factores asociados al uso de terapias de MAC, a la edad, sexo, estado civil, grado de instrucción, ocupación e ingreso económico.

\section{Procedimiento y recolección de datos}

El instrumento utilizado consistió en una ficha de recolección de datos de 24 preguntas de opción múltiple, dividida en dos secciones: datos generales, en donde se recolectó información sobre los posibles factores socioepidemiológicos asociados al uso de MAC; $y$ aspectos relacionados al uso de MAC en la población (Material suplementario), donde se observa el uso de estas terapias.

Se indagó sobre el uso de quince terapias distintas: acupuntura, fitoterapia/medicina herbal, homeopatía, terapia neural, trofoterapia, aromaterapia, quiropraxia, masoterapia, reflexoterapia, yoga, tai chi, hidroterapia, geoterapia, reiki, terapia magnética $\mathrm{y}$ «otros» donde se incluían otras terapias 
no disponibles en la lista. La selección de estas terapias se realizó en función a estudios previos ${ }^{(10)}$, referencias de otras instituciones prestadoras de salud que brindan servicios de medicina complementaria ${ }^{(1)} \mathrm{y}$ al juicio de dos expertos en investigación en MAC y en aspectos de interculturalidad.

El grupo de investigación capacitó a encuestadores externos en tópicos básicos de terapias de MAC y en la aplicación del instrumento. Se realizó juicio de expertos y ensayos pilotos previos a la ejecución del estudio para mejorar el entendimiento y el tiempo en la aplicación del instrumento. El grupo de investigación supervisó directamente el trabajo de campo.

\section{Análisis de datos}

Se utilizó estadística descriptiva (frecuencias y porcentajes) para mostrar la distribución de las variables de estudio en las personas incluidas en la investigación. Posteriormente, se realizó un análisis bivariado para corroborar la presencia de asociación estadística entre cada uno de los posibles factores asociados y el uso de MAC, mediante la prueba Chi cuadrado. Estas pruebas se realizaron con la ayuda del programa STATA versión 13 (StataCorp. 2013. Stata Statistical Software: Release 13. College Station, TX: StataCorp LP).

\section{Aspectos éticos}

Esta investigación fue aprobada por el Comité de Ética en Investigación del Instituto Nacional de Salud. Debido a su naturaleza, fue un estudio de mínimo riesgo para los participantes y se solicitó su consentimiento informado antes de ser incluidos. No se obtuvieron datos que permitieran la identificación de las personas encuestadas.

\section{HALLAZGOS}

Durante el trabajo de campo, se aplicaron 917 encuestas en los tres distritos seleccionados: 509 (55,5\%), en Callería; 232 (25,3\%), en Manantay; y 176 (19,2\%), en Yarinacocha. La mayoría de los encuestados fueron mujeres $(68,3 \%)$ y se encontraron primordialmente en el rango de edades de 18 a 30 años $(29,2 \%)$. Adicionalmente, un $38,6 \%$ se dedicaba al cuidado del hogar y un $79,9 \%$ tenía ingresos económicos menores a 1436 soles mensuales (Tabla 1 ).

179 encuestados $(19,5 \%)$ mencionan que utilizaron la MAC en los últimos doce meses, de los cuales, $36(20,1 \%)$ refieren que utilizaron estos métodos junto con terapias convencionales y solo $22(12,2 \%)$ informaron a su médico tratante que estaban utilizando terapias de MAC.

El 68,7\% de las personas que utilizaron la MAC refieren que acudieron a establecimientos privados (particulares), mientras que el 20,7\% acudió al Seguro Social de Salud (EsSalud). Un menor porcentaje acudió a organizaciones religiosas (3,5\%),
Tabla 1. Características de las personas encuestadas $(n=917)$ en tres distritos de la provincia de Coronel Portillo (Ucayali) en 2013

\begin{tabular}{|c|c|}
\hline Características & n (\%) \\
\hline \multicolumn{2}{|l|}{ Sexo } \\
\hline Femenino & $626(68,3)$ \\
\hline Masculino & $291(31,7)$ \\
\hline \multicolumn{2}{|l|}{ Edad (años) } \\
\hline $18-30$ & $268(29,2)$ \\
\hline $31-40$ & $183(19,9)$ \\
\hline $41-50$ & $176(19,1)$ \\
\hline $51-60$ & $162(17,8)$ \\
\hline $61-70$ & $92(10,0)$ \\
\hline 71 a más & $36(3,9)$ \\
\hline \multicolumn{2}{|l|}{ Estado civil } \\
\hline Conviviente & $367(40,0)$ \\
\hline Soltero/a & $252(27,5)$ \\
\hline Casado/a & $216(23,6)$ \\
\hline Viudo/a & $46(5,0)$ \\
\hline Separado/a & $25(2,7)$ \\
\hline Divorciado/a & $11(1,2)$ \\
\hline \multicolumn{2}{|l|}{ Grado de instrucción } \\
\hline Secundaria & $429(46,8)$ \\
\hline Primaria & $199(21,7)$ \\
\hline Superior completa & $172(18,8)$ \\
\hline Superior incompleta & $109(11,9)$ \\
\hline Sin instrucción & $8(0,9)$ \\
\hline \multicolumn{2}{|l|}{ Ocupación } \\
\hline Su casa & $354(38,6)$ \\
\hline Independiente & $349(38,1)$ \\
\hline Empleado público & $98(10,7)$ \\
\hline Empleado privado & $59(6,4)$ \\
\hline Estudiante & $48(5,2)$ \\
\hline Agricultura y ganadería & $9(0,9)$ \\
\hline \multicolumn{2}{|l|}{ Ingreso económico (soles) } \\
\hline$<1436$ & $733(79,9)$ \\
\hline $1436-2045$ & $145(15,8)$ \\
\hline $2046-3376$ & $32(3,5)$ \\
\hline $3377-5308$ & $2(0,2)$ \\
\hline$>5308$ & $5(0,5)$ \\
\hline \multicolumn{2}{|c|}{ Uso de MAC en los últimos 12 meses } \\
\hline Sí & $179(19,5)$ \\
\hline No & $738(80,4)$ \\
\hline
\end{tabular}

MAC: medicina alternativa y complementaria

a establecimientos del Ministerio de Salud (0,6\%), entre otros $(3,5 \%)$. Al indagar por los motivos que tuvieron los encuestados para utilizar la MAC, un 45,8\% mencionó que lo hacía por recomendación de un familiar o amigo; un 15,6\% refirió 
usarla debido a que la medicina convencional no le brindaba solución a su problema de salud; y un 13,9\% consideraba que estas terapias son eficaces.

La terapia de MAC más utilizada por los encuestados fue fitoterapia $(62,0 \%)$; seguido de acupuntura $(17,9 \%)$, tai chi $(10,1 \%)$, trofoterapia $(8,9 \%)$, reflexoterapia $(7,3 \%)$ y masoterapia $(5,6 \%)$. Otros métodos reportados fueron quiropraxia, yoga, geoterapia y, en menor cantidad, terapias magnéticas, hidroterapia y homeopatía (Figura 1).

Al realizar el análisis bivariado, se encontró una relación estadísticamente significativa entre el uso de terapias de MAC y el grado de instrucción ( $\mathrm{p}<0,001)$, la ocupación de la persona encuestada $(\mathrm{p}<0,001)$ y el ingreso económico mensual $(\mathrm{p}<0,001)$ (Tabla 2).

\section{DISCUSIÓN}

Se encontró una frecuencia de uso de terapias de MAC de un $19,5 \%$, siendo la fitoterapia o uso de plantas medicinales la más utilizada. Estos resultados son coherentes con lo encontrado por Mejía Gálvez et al. en un estudio realizado en pacientes de consulta externa en Lima Metropolitana, donde se encontró que la fitoterapia fue utilizada en un $29,6 \%{ }^{(6)}$, y por Gamarra Condezo, quien reportó una frecuencia de uso de 24,7\% en Huánuco ${ }^{(12)}$. Medina Larico encontró 69 especies de plantas utilizadas para fines medicinales en la comunidad nativa Nuevo Saposoa, en la misma provincia en la que se realizó el presente estudio ${ }^{(13)}$. Adicionalmente, Chuspe Zans también evidenció que se preserva el conocimiento ancestral sobre el uso de plantas originarias en la etnia Uni de la provincia de Aguaytia ${ }^{(14)}$ y en la etnia shipiba del distrito de Masisea (provincia de Coronel Portillo) ${ }^{(15)}$.

En el análisis bivariado se encontró asociación estadística entre la ocupación, el ingreso económico, y el grado de instrucción. En el caso de los factores «ocupación» e «ingreso económico», los estudios previos han mostrado resultados contradictorios. La revisión sistemática de Bai James et al. resume los hallazgos de diez estudios en los que se en-

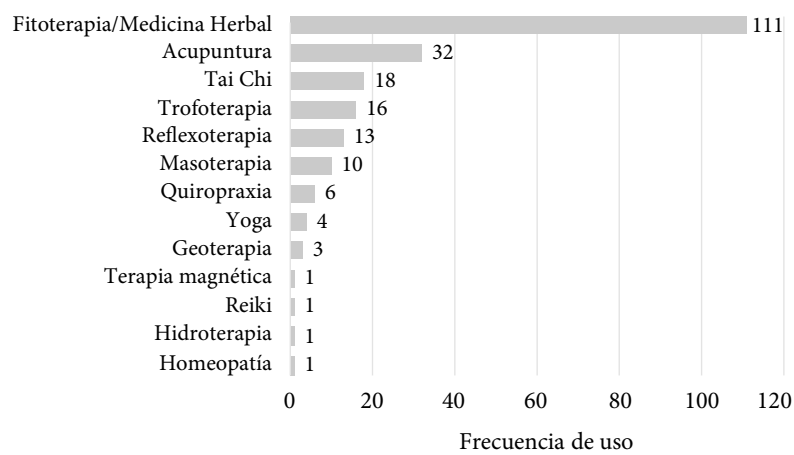

Figura 1. Distribución de terapias de medicina alternativa y complementaria utilizadas en tres distritos de la provincia de Coronel Portillo (Ucayali) en 2013
Tabla 2. Factores asociados al uso de la medicina alternativa y complementaria en tres distritos de la provincia de Coronel Portillo (Ucayali) en 2013

\begin{tabular}{|c|c|c|c|}
\hline \multirow[t]{2}{*}{ Variables } & \multicolumn{2}{|c|}{$\begin{array}{c}\text { Uso de MAC } \\
(\mathbf{n}=917)\end{array}$} & \multirow{2}{*}{$\begin{array}{l}\text { Valor } \\
\text { de } p^{a}\end{array}$} \\
\hline & Sí & No & \\
\hline Sexo & & & 0,655 \\
\hline Femenino & $54(18,6)$ & $237(81,4)$ & \\
\hline Masculino & $125(20,0)$ & $501(80,0)$ & \\
\hline Edad (años) & & & 0,604 \\
\hline $18-30$ & $48(17,9)$ & $220(82,1)$ & \\
\hline $31-40$ & $34(18,6)$ & $149(81,4)$ & \\
\hline $41-50$ & $37(21,0)$ & $139(79,0)$ & \\
\hline $51-60$ & $37(22,8)$ & $125(77,1)$ & \\
\hline $61-70$ & $19(20,6)$ & $73(79,4)$ & \\
\hline 71 a más & $4(11,1)$ & $32(88,9)$ & \\
\hline Estado civil & & & 0,081 \\
\hline Soltero/a & $59(23,4)$ & $193(76,6)$ & \\
\hline Casado/a & $50(23,1)$ & $166(76,9)$ & \\
\hline Viudo/a & $8(17,4)$ & $38(82,6)$ & \\
\hline Separado/a & $4(16,0)$ & $21(84,0)$ & \\
\hline Divorciado/a & $3(27,3)$ & $8(72,7)$ & \\
\hline Grado de instrucción & & & $<0,001$ \\
\hline Sin instrucción & $0(0,0)$ & $8(100,0)$ & \\
\hline Primaria & $24(12,1)$ & $175(87,9)$ & \\
\hline Secundaria & $71(16,5)$ & $358(83,5)$ & \\
\hline Superior incompleta & $23(21,1)$ & $86(78,9)$ & \\
\hline Superior completa & $61(35,5)$ & $111(64,5)$ & \\
\hline Ocupación & & & $<0,001$ \\
\hline Su casa & $51(14,4)$ & $303(85,6)$ & \\
\hline Independiente & $68(19,5)$ & $281(80,5)$ & \\
\hline Empleado público & $31(31,6)$ & $67(68,4)$ & \\
\hline Empleado privado & $18(30,5)$ & $41(69,5)$ & \\
\hline Estudiante & $11(22,9)$ & $37(77,1)$ & \\
\hline Agricultura y ganadería & $0(0,0)$ & $9(100,0)$ & \\
\hline Ingreso económico (soles) & & & $<0,001$ \\
\hline$<1436$ & $121(16,5)$ & $612(83,5)$ & \\
\hline $1436-2045$ & $45(31,0)$ & $100(69,0)$ & \\
\hline $2046-3376$ & $9(28,1)$ & $23(71,9)$ & \\
\hline $3377-5308$ & $2(100,0)$ & $0(0,0)$ & \\
\hline$>5308$ & $2(40,0)$ & $3(60,0)$ & \\
\hline
\end{tabular}

MAC: medicina alternativa y complementaria

a Prueba de Chi cuadrado

contró relación estadística entre el uso de la MAC, un estado socioeconómico bajo y el no tener empleo formal (o ser un empleado independiente) ${ }^{(16)}$. Estos hallazgos son coherentes con el estudio de Peltzer y Pengpid, quienes encontraron una relación entre los indicadores socioeconómicos bajos y 
el uso de terapias de MAC ${ }^{(17)}$. Sin embargo, los estudios de Wemrell et al. y Johny et al. no encontraron asociación significativa entre el consumo de terapias de MAC y el nivel socioeconómico en usuarios de Suecia ${ }^{(18)}$ y de Malasia ${ }^{(19)}$, respectivamente.

En forma similar al presente estudio, Abdullah et al. encontraron que un nivel educativo superior (técnico o universitario) se encontraba relacionado a una mayor utilización de terapias de MAC en Malasia ${ }^{(20)}$. Sin embargo, aún existen estudios que no encuentran relación entre el nivel educativo y el uso de MAC ${ }^{(19)}$, incluso encuentran que la educación universitaria es un factor protector ante el uso de estas terapias ${ }^{(17,20)}$. Sin embargo, a diferencia de este estudio, las investigaciones previas no utilizan muestreos probabilísticos para la selección de las personas encuestadas, por lo que esta investigación sí permite establecer conclusiones que puedan ser muy similares a lo que ocurre en el ámbito de la población estudiada.

Dentro de las limitaciones de este estudio se debe mencionar que la utilización de instrumentos de recolección de datos como fichas, implican la probabilidad inherente de un sesgo de información y un sesgo de memoria por parte de los encuestados. Por otro lado, el hecho de no considerar el uso de un muestreo complejo altera los estimados finales, y la antigüedad de los datos podría repercutir en que estos hallazgos ya no sean aplicables en la actualidad. Sin embar-

\section{REFERENCIAS BIBLIOGRÁFICAS}

1. Organizacion Mundial de la Salud. Estrategia de la OMS sobre Medicina Tradicional (2014-2023). 1. a ed. Hong Kong: Organizacion Mundial de la Salud; 2013. Disponible en: http://apps.who.int/medicinedocs/ es/m/abstract/Js21201es/.

2. Gannotta R, Malik S, Chan AY, Urgun K, Hsu F, Vadera S. Integrative Medicine as a Vital Component of Patient Care. Cureus. 10(8). doi: 10.7759/cureus.3098.

3. Caceres Guido P, Ribas A, Gaioli M, Quattrone F, Macchi A. The state of the integrative medicine in Latin America: The long road to include complementary, natural, and traditional practices in formal health systems. Eur J Integr Med. 2015;7(1):5-12. doi: 10.1016/j. eujim.2014.06.010.

4. Wardle J, Lui C-W, Adams J. Complementary and alternative medicine in rural communities: current research and future directions. J Rural Health. 2012;28(1):101-12. doi: 10.1111/j.17480361.2010.00348.x.

5. Ray J, Chakrabarty D, Paul R, Som K. Prevalence of the use of complementary and alternative medicine in an eastern Indian population with emphasis on tribal/ethnic minority groups. J Taibah Univ Med Sci. 2018;13(4):384-9. doi: 10.1016/j.jtumed.2018.04.001.

6. Mejía Gálvez JAM, Carrasco E, Miguel JL, Flores SA. Conocimiento, aceptación y uso de medicina tradicional peruana y de medicina alternativa/ complementaria en usuarios de consulta externa en Lima Metropolitana. Rev Peru Med Integrativa. 2017;2(1):47-57. doi: 10.26722/rpmi.2017.21.44

7. Ministerio de Salud. Análisis de Situación de Salud del Perú. 2018. Lima: Centro Nacional de Epidemiología, Prevención y Control de Enfermedades, MINSA; 2018. [citado el 17 de julio de 2019]. Disponible en: https://www.dge.gob.pe/portal/docs/asis/Asis_peru19.pdf. go, los resultados de este estudio son relevantes por la falta de información sobre el uso de la MAC en la población amazónica. Futuros estudios, deberán plantear otros tipos de muestreo y diseños prospectivos para controlar, en lo posible, estas limitaciones.

Adicionalmente, no se ahondó en aspectos como la composición familiar o si la persona que brindaba la información era o no el jefe de familia; por lo que se recomienda que posteriores investigaciones agreguen estos aspectos en los instrumentos de recolección de datos.

Se concluye que, casi la quinta parte de la muestra estudiada utilizaron terapias de MAC en los últimos 12 meses, ademas el grado de instrucción, la ocupación y los ingresos económicos resultaron ser factores asociados al uso de estas terapias. Esta investigación brinda información relevante sobre la situación del uso de MAC por la población amazónica, en su contexto sociocultural y dinámica socioeconómica.

Contribuciones de autoría: RSA y FVO concibieron y diseñaron el estudio. RSA y FVO recolectaron datos e información. RSA, FVO y YAB redactaron el artículo, y analizaron e interpretaron los resultados. RSA y FVO obtuvieron el financiamiento. Todos los autores participaron en la revisión crítica y aprobación de la versión final.

Fuente de financiamiento: Instituto Nacional de Salud, Lima, Perú.

Conflictos de interés: Los autores declaran no tener conflictos de interés.

Material suplementario: Disponible en la versión electrónica de la RPMESP.
8. Instituto Nacional de Estadistica e Informatica (INEI). Censos Nacionales 2007: XI de Población y VI de Vivienda. Lima: Instituto Nacional de Estadística e Informática; 2008.

9. Municipalidad de Coronel Portillo. Plan de desarrollo concertado provincial de Coronel Portillo 2009-2021. Coronel Portillo; 2009.

10. Santiváñez R., Yagui M. Perú: Medicina Alternativa y Medicina Complementaria. Lima: Dirección Ejecutiva de Medicina Alternativa y Complementaria, Instituto Nacional de Salud; 2012 [citado el 17 de julio de 2019]. Disponible en https://www.ins.gob.pe/repositorioaps/0/7/jer/censi_prom_met/2015/brochure.pdf.

11. Gerencia Central de Atención Primaria. Normas para la Atención de Medicina Complementaria en los Servicios del Primer Nivel de Atención de EsSalud. EsSalud; 2013.

12. Gamarra Condezo N. Usos de plantas medicinales por usuarios externos del Hospital Regional Hermilio Valdizan Medrano-Huanuco, 2016. [Tesis para obtener el título profesional de licenciada en Enfermeria]. Huanuco: Universidad de Huanuco; 2017 [citado el 17 de julio de 2019]. Disponible en: http://localhost:8080/xmlui/ handle/123456789/651.

13. Medina Larico, Amparo. Etnobotánica cuantitativa de las plantas medicinales en la Comunidad Nativa Nuevo Saposoa, Provincia Coronel Portillo, Ucayali-Perú [Tesis para optar por el título profesional de Biología]. Arequipa: Universidad Nacional de San Agustín; 2018 [citado el 17 de julio de 2019]. Disponible en: http://repositorio.unsa. edu.pe/handle/UNSA/5087.

14. Chuspe Zans M. Uso de plantas medicinales en el tratamiento de enfermedades y conservación de la salud en los uni: Provincia de Aguaytía, Region de Ucayali - Perú. Dans: Etnobotanica y Fitoterapia en America. Brno: Universidad de Mendel; 2015. p. 164-98. 
15. Cauper S. Estudio de plantas medicinales desde conocimientos shipibo. Masisea, Perú. Cienc Desarro. 2019;21(2):07-26. doi: 10.21503/cyd.v21i2.1705.

16. James PB, Wardle J, Steel A, Adams J. Traditional, complementary and alternative medicine use in Sub-Saharan Africa: a systematic review. BMJ Glob Health. 2018;3(5):e000895. doi: 10.1136/bmjgh-2018-000895.

17. Peltzer K, Pengpid S. Prevalence and Determinants of Traditional, Complementary and Alternative Medicine Provider Use among Adults from 32 Countries. Chin J Integr Med. 2018;24(8):584-90. doi: 10.1007/ s11655-016-2748-y.

18. Wemrell M, Merlo J, Mulinari S, Hornborg A-C. Two-Thirds of Survey Respondents in Southern Sweden Used Complementary or Alterna- tive Medicine in 2015. Complement Med Res. 2017;24(5):302-9. doi: $10.1159 / 000464442$.

19. Johny AK, Cheah WL, Razitasham S. Evidence-Based Complementary and Alternative Medicine [Internet]. 2017. Disclosure of Traditional and Complementary Medicine Use and Its Associated Factors to Medical Doctor in Primary Care Clinics in Kuching Division, Sarawak, Malaysia. [citado el 18 de julio de 2019]. Disponible en https://www. hindawi.com/journals/ecam/2017/5146478/.

20. Abdullah N, Borhanuddin B, Patah AEA, Abdullah MS, Dauni A, Kamaruddin MA, et al. Utilization of Complementary and Alternative Medicine in Multiethnic Population: The Malaysian Cohort Study. J Evid-Based Integr Med. 2018;23:2515690X18765945. doi: 10.1177/2515690X18765945. 